\title{
Las Nuevas Tecnologías en el Proyecto de Desarrollo Institucional de la Universidad Distrital Francisco José de Caldas
}

\author{
José Nelson Pérez Castillo, Blanca Magdalena Castro Vargas \\ Facultad de Ingeniería \\ Universidad Distrital Francisco José de Caldas \\ Carrera 8 No 40 - 68, Piso 3 \\ Bogotá, Colombia \\ Teléfono: 571- 3271025 \\ Fax: 571 - 3271026 \\ Email: \{nelsonp, magdacas\}@udistrital.edu.co
}

\begin{abstract}
Resumen
Tres grandes desafíos que se plantean a la educación superior en el presente milenio son: pertinencia, calidad e internacionalización. Indudablemente, estos tres requerimientos, encuentran un soporte fundamental en las modernas tecnologías de la información, las comunicaciones y la gestión del conocimiento, según se muestra en este artículo.
\end{abstract}

Palabras Clave : portal de conocimiento, redes multimedia, sistemas de información, campus inteligente

Abstract

Three challenging purposes which are to be taken into account when talking about undergraduate education present millennium. They are: relevancy, quality and internationalization. Undoubtedly, these three requeriments are well supported in the modern technologies of information, communications and knowledge management, as shown in this paper.

Key words : knowledge portal, multimedia networks, information systems, intelligent campus

\section{Introducción}

La universidad como ente educativo que busca ponerse a tono con el proceso de modernización mundial según los requerimientos de pertinencia, calidad e internacionalización, debe revisar y revaluar sus métodos de enseñanza y ajustar sus currículos a la nueva situación. Esto hace urgente el replanteamiento del quehacer universitario, buscando la formación de pensadores en comunidades disciplinarias, autónomos, racionales, participativos y responsables, capaces de hacer ciencia dentro de sus profesiones, teniendo en cuenta que esto se logra con la investigación especializada y la utilización de suficientes recursos tecnológicos puestos a su alcance.

La educación superior, vista así, resulta ser un factor fundamental del desarrollo; pero, para que esto no sea sólo una frase más, es necesario incorporarle plenamente los beneficios de las nuevas tecnologías, que permiten tener acceso a nuevas formas de difusión y apropiación del conocimiento, propiciando la formación de hombres innovadores y creativos, capaces de participar en la transformación que exige la rapidez del desarrollo. 
Esto reclama obviamente la presencia en la universidad de nuevas metodologías y modelos pedagógicos, en los cuales se privilegie la investigación, la interacción y el trabajo en equipo, situación que exige la dotación de una infraestructura adecuada, capaz de dar cobertura a todas las dependencias de la institución y la capacitación y desarrollo de habilidades para la óptima utilización de dichos recursos. La internacionalización de los conocimientos implica la superación de métodos anacrónicos de investigación y la disponibilidad de los instrumentos de comunicación que hagan posible una mayor integración de las comunidades educativas, académicas y científicas.

A pesar de reconocer de plano, la importancia preponderante en la educación de las nuevas iniciativas pedagógicas y su estrecha relación con la ciencia cognitiva, la enseñanza y el aprendizaje, en este documento, se opta por presentar consideraciones científicas y tecnológicas, que desde la visión de un grupo de profesores de la Universidad Distrital, resultan también de importancia crítica para la educación superior en el siglo XXI. En tal sentido, las nuevas tecnologías de intercomunicación y difusión introducen de manera creciente, importantes oportunidades en términos de interacción asincrónica entre protagonistas del proceso de creación y difusión del conocimiento, al tiempo que contribuye a democratizar el acceso a sistemas de información distribuida, disminuyendo el control del conocimiento por determinados grupos.

La necesidad de vincular la universidad al desarrollo social, requiere entre otras cosas, sistemas de comunicaciones modernos con cubrimiento institucional generalizado a todos sus estamentos y disciplinas, partiendo del convencimiento que la vía para la reducción de la pobreza mental y material, el subdesarrollo y la dependencia, es el cultivo de una práctica universitaria basada en una tecnología científica, robusta y fértil que permita desarrollar el pensamiento humano para el manejo y la transformación de la cultura en beneficio social e individual.

En la actualidad las tecnologías de información, comunicaciones y gestión del conocimiento [Schreiber, 2000] son decisivas para el uso compartido y transferencia de información y conocimientos entre instituciones [Silvio, 2000]. De hecho, la tendencia creciente hacia el uso intensivo de la computación en las diferentes esferas de la sociedad es una consecuencia directa de la interconexión y la disponibilidad de recursos informáticos y de comunicaciones en gran escala.

\section{Proyectos de Investigación y Desarrollo}

En seguida se esbozan someramente los principales proyectos de orden institucional que implican la inserción en el plan de desarrollo de avanzadas tecnologías de información, comunicaciones y gestión de conocimiento.

\section{Portal de Conocimiento}

Un portal de conocimiento, es un portal mejorado de información cuyo objetivo es la producción, integración y gestión de conocimiento [Firestone, 2000]. De hecho produce, provee y gestiona información con respecto a la validez de la información misma que suministra. Además, se distingue de la mera información, provee facilidades para la generación de conocimiento a partir de información y se orienta hacia la producción e integración de conocimiento más que de información [Nonaka, 1995]. Puede de una vez decirse, que un portal de conocimiento es una aplicación que sitúa en la Web, la producción, la integración y la gestión de conocimiento.

Dado que un portal de conocimiento comparte características de los portales de información, incorpora una interfaz con base en un navegador, gestiona datos 
estructurados y contenidos no estructurados, como también producción, integración y gestión cooperativas de conocimiento. Esto significa que los casos de uso [Booch, 1999], deben soportar la adquisición de información, el aprendizaje individual y en grupo, la formulación y validación de demandas de conocimiento, la difusión, búsqueda y recuperación, el uso compartido y la enseñanza. En cada una de estas áreas, el portal de conocimiento soporta la planeación, el monitoreo y la evaluación de los ciclos de decisión.

Los portales de conocimiento distinguen conocimiento de información, al proporcionar información referente a los resultados de las pruebas de validez de cualquier porción de información. Esto significa, que los portales de conocimiento deben almacenar metainformación que dice del grado de confiabilidad en el conocimiento demandado frente al de sus competidores, es decir, los portales de conocimiento registran la historia de la lucha competitiva entre las ideas puestas en concurso para resolver problemas dentro del ámbito organizacional.

Un portal de conocimiento en un entorno universitario, es un sistema de tecnologías avanzadas dirigido hacia la producción, adquisición, transmisión y gestión de conocimiento centrado en las actividades educativas, de investigación y de gestión académica. Además, tal portal de conocimiento debe ser capaz de proveer, producir y gestionar información con respecto al conocimiento e información que suministra.

En otras palabras, un portal de conocimiento en el ámbito universitario, provee información con respecto a las diferentes actividades de la institución pero adicionalmente proporciona metainformación como un ingrediente fundamental para la toma de decisiones. Por lo tanto, un portal de conocimiento, es capaz de discriminar entre los conceptos de conocimiento e información. Esto quiere decir, que facilita la producción de conocimiento a partir de datos e información. De ahí que un portal de conocimiento, se orienta más a la producción, adquisición y transmisión de conocimiento que a la sola información. Entonces, intrínsecamente proveen un mejor fundamento para la toma de decisiones que los portales de información. Quienes tienen conocimiento, tienen una clara ventaja competitiva sobre quienes tienen únicamente información.

Los portales de conocimiento representan una solución a este reto, puesto que proveen un entorno flexible de conocimiento a una cantidad potencialmente enorme de usuarios. La misión de un portal de conocimiento no es sólo facilitar el acceso a un gran volumen de información similar al de una biblioteca, sino ayudar activamente a los interesados en sus procesos académicos, científicos y culturales.

El portal de conocimiento proveerá un mecanismo de acceso a la dinámica de gestión del conocimiento en la Universidad Distrital, con base en la Web, de manera personalizada, permitiendo a los distintos usuarios (estudiante, docentes, administrativos, ciudadanos, sector gubernamental) un visión personalizada de las respuestas a sus requerimientos de información y conocimiento.

\section{Universidad Distrital Virtual}

Se concibe la Universidad Virtual como aquella institución de educación superior que soporta sus actividades administrativas, educativas y de investigación, superando las fronteras del tiempo y el espacio. Los conceptos de aprendizaje asíncrono y clase virtual como el estudio y la investigación en torno a los modelos pedagógicos más adecuados hacen parte de la idea de universidad virtual. No obstante, este entorno educativo, debe apoyarse en una adecuada infraestructura de sistemas informáticos, de comunicaciones y de gestión del conocimiento. 
Según esto, el proyecto de Universidad Distrital Virtual (UDV), como se le ha denominado hasta ahora, se constituye en un auténtico proyecto innovativo de investigación y desarrollo. Esto significa, que es necesario desagregar esta iniciativa en tópicos de investigación que contribuyan a dar pasos fundamentales en la aplicación de tecnologías de integración de información fundamentales y en la consolidación y desarrollo de los componentes de la universidad virtual.

Los estudios e investigaciones relacionados a las tecnologías de información incluyen la integración de metodologías orientadas a objetos [Meyer, 1988], [D’Souza, 1999], [Booch, 1996a], bajo el paradigma de sistemas distribuidos en la Web [Ginige, 2001a], [Ginige, 2001b] y la definición de esquemas de seguridad en los campos de Internet e Intranet.

La integración de los diversos componentes de la UDV exige la modernización de sistemas de soporte administrativo tales como los de control académico, de personal, financiero, de investigación y de gestión del flujo de trabajo y el desarrollo de otros subsistemas tales como publicaciones electrónicas, biblioteca digital y sistemas de gestión en línea.

Desde el punto de vista tecnológico, es preciso enfocarse en el desarrollo de infraestructuras de información, seguridad en bases de datos, redes de comunicaciones y facilidades de hardware como también la implementación de sistemas de flujo de trabajo y de comunicación básicos por encima de las facilidades acumuladas de hardware y bases de datos. De ahí, que sea imprescindible reflexionar sobre una arquitectura que permita modelar el ensamblaje de los distintos componentes de la UDV.

\section{Campus Inteligente De La Universidad Distrital}

La iniciativa de campus inteligente, comenzará con la aplicación del concepto de edificios inteligentes en la Universidad Distrital, especialmente para aquellos actualmente en construcción. Al respecto existen tecnologías que hacen posible tal implementación y constituyen el fundamento de la iniciativa para la puesta en marcha del campus inteligente.

Las actuales posibilidades tecnológicas permiten ofrecer comodidad, seguridad, confiabilidad, flexibilidad y eficiencia de manera acorde con el planteamiento básico de una educación de calidad que garantice la equidad social [Cárdenas, 2001].

La comodidad se traduce en las funciones que permiten tener mejores condiciones de actuación y de trabajo (control ambiental), tales como iluminación adecuada y climatización entre otras.

La seguridad es un aspecto básico que involucra las funciones de protección del patrimonio del recurso humano tales como control de ingreso y estacionamiento de vehículos, detección, alarma y combate de incendios; detección y control de actos de intrusión; monitoreo de imágenes; sistemas de intercomunicación. Se puede estar en capacidad de situar el monitoreo de cada parámetro de la universidad en sitios en los niveles local, de campus, metropolitano, nacional o internacional haciendo uso de la red académica UDNET, adicionando así valor agregado a ella.

La confiabilidad es un factor específico de seguridad relacionado con el buen funcionamiento de los equipos y sistemas de infraestructura. Para ello se monitorea con equipos de óptima calidad el funcionamiento de cada subsistema como energía eléctrica, 
hidráulica y de gas, fuentes alternas y dispositivos de reserva. Adicionalmente se implementan sistemas administrativos de mantenimiento.

La flexibilidad significa que el sistema instalado es diseñado para aceptar nuevos recursos tecnológicos, equipos, sistemas y funciones. De la misma forma estará en capacidad de aceptar las tecnologías que corrientemente estén siendo usadas para que cumplan su ciclo de vida útil.

La eficiencia tiene que ver con el uso racional de los recursos para lograr un funcionamiento al menor costo con el máximo logro. Por tanto el sistema provee manejo energético y control de procesos.

\section{Red Multimedia UDNET}

\section{Backbone Metropolitano de Fibra Óptica}

Está infraestructura física de la Universidad Distrital estará constituida por un anillo de fibra óptica de modo que se enlacen las actuales y futuras sedes de la institución. El diseño, desarrollo y puesta en marcha de este proyecto reivindica la tradición académica de la Facultad de Ingeniería de la universidad, gracias al trabajo de largos años en la línea de investigación en optolectrónica y a la formación que se imparte en el postgrado de Maestría en Teleinformática.

Se busca, dotar a la Universidad Distrital de una auténtica superautopista de información que haga posible disponer del ancho de banda necesario requerido por las aplicaciones multimediales que serán el fundamento para la pedagogía de la interactividad en la futura universidad virtual.

Esto, facilitará una adecuada conformación del tráfico multimedia en una universidad completamente insertada en la sociedad del conocimiento. Además, se dispondrá de los anchos de banda necesarios que permitan acomodar las distintas aplicaciones pedagógicas de una auténtica universidad preparada para la sociedad del conocimiento, entendida como aquella, que se construye en torno a la comunicación aspecto fundamental y decisivo en la educación.

\section{Red Metropolitana ATM}

Los requerimientos educativos de la sociedad actual están cambiando a un ritmo no imaginado. La educación se ha convertido en un proceso para toda la vida, que permite a las personas en sus distintos campos mantenerse a tono con los cambios en sus distintas disciplinas. Para satisfacer estas necesidades las instituciones universitarias deben ir más allá de las nociones convencionales de campus para ofrecer servicios educativos a sus estudiantes.

Durante los últimos años se han producido cambios radicales en la tecnología de las telecomunicaciones, particularmente en sistemas de transmisión y dispositivos ópticos. Las redes operan en el orden de los gigabits por segundo. Al mismo tiempo, existen procesadores poderosos y dispositivos de almacenamiento de alta capacidad que facilitan el surgimiento cada vez mayor de aplicaciones multimediales. Entonces, existe la oportunidad de usar estos desarrollos construyendo un nuevo ambiente para la educación virtual que tome ventaja del poder computacional de la llamada multimedia y de la capacidad expandida de las redes, facilitando a grupos de usuarios, ubicados en diversos puntos geográficos, comunicarse y compartir documentos multimediales para mejorar los procesos de aprendizaje. 
Además, la Universidad Distrital necesita construir la infraestructura necesaria para ofrecer los escenarios de aprendizaje que exige el proyecto de universidad virtual, en particular los ambientes de aprendizaje a distancia, que deberá traducirse en una mayor calidad de los procesos educativos a menor costo, para los distintos actores distribuidos en el área metropolitana del Distrito Capital.

Con fundamento en las capacidades de una red ATM, la Universidad Distrital se orienta al estudio, desarrollo e implementación de un sistema que haga posible un entorno multimedial que permita el acceso a los distintos miembros de la comunidad académica desde cualquier lugar y en cualquier momento. Este proyecto implica el poder ofrecer a los residentes en el área metropolitana de Santa Fe de Bogotá, clases interactivas que incluyan audio, video e imágenes de alta resolución sobre una red ATM, con las consiguientes ventajas que ello implica.

Se ofrecerá a la comunidad del Distrito Capital en general y a la comunidad académica de la Universidad Distrital en particular, una red multimedios acorde con las exigencias de la sociedad del conocimiento. De paso esto favorece el desarrollo del proyecto de universidad virtual, contribuye a la construcción del campus inteligente de la Universidad Distrital, e impulsa los procesos educativos según las exigencias de la postmodernidad.

\section{Telepuerto}

Dentro de la iniciativa del campus inteligente, se busca dotar a la universidad, de un telepuerto, que facilite acceso al segmento espacial centrado en la formación, creación y difusión de materiales didácticos audiovisuales vía satélite al medio iberoamericano.

La Universidad Distrital tiene especial interés en la difusión de materiales educativos de televisión y en el papel que en esta última puedan desempeñar las telecomunicaciones. Quiere esto decir, que a pesar de ser las experiencias educativas distribuidas vía satélite el motivo principal del proyecto, no constituye su único interés, abarcando su campo de actuación todas aquellas experiencias e innovaciones educativas apoyadas en las telecomunicaciones.

Los continuos avances en el mundo de las telecomunicaciones muestran un panorama en constante evolución. Este escenario cambiante, puede constituir, quizá, el principal aliciente para el interés de la Universidad Distrital en constituirse en proveedor de segmento espacial gracias a su telepuerto privado. Este proyecto implica la consideración y ponderación de tres aspectos:

1. La producción de materiales educativos. Dentro de lo que puede entenderse como aplicaciones educativas de la televisión o, también, televisión educativa, suelen diferenciarse tres tipos: la televisión cultural, con objetivos de divulgación y entretenimiento, la televisión educativa propiamente dicha, de contenidos extracurriculares, pero con objetivos relacionados con la formación, y la televisión escolar, con contenidos y objetivos propios del sistema educativo. Cada uno de estos tipos requiere modalidades distintas de producción, y a su vez cada uno de ellos ofrece posibilidades diversas para la Universidad Distrital.

2. La distribución de experiencias de formación vía satélite. Puede decirse que las experiencias de distribución de la formación vía satélite, todavía, son escasas. No obstante, es previsible un desarrollo importante en las prestaciones y en la capacidad del segmento espacial, desarrollo que permitirá promover nuevas experiencias educativas. Esta mayor capacidad vendrá dada, por una parte, por el 
aumento del número de satélites en órbita, que junto a la liberalización de las comunicaciones vía satélite, proporcionará múltiples posibilidades a la educación a distancia basada en satélites; y por otra parte, el creciente grado de sofisticación tecnológica y un mayor aprovechamiento de los satélites, propiciando el crecimiento de experiencias y proyectos educativos.

3. Las innovaciones en los sistemas de comunicación. De los avances que en las tecnologías de la comunicación se producen día a día, quizá sean las posibilidades de las redes y la televisión por cable las que mayor impacto pueden tener sobre el ámbito de la formación. La Universidad Distrital entiende las posibilidades que estas tecnologías aportan a las experiencias de formación. Puede decirse que las redes, además de proporcionar variadas posibilidades comunicativas, configuran nuevos sistemas de enseñanza que, entre las modalidades, presencial y virtual, suponen profundos cambios en la estructura y organización de dichos sistemas. De esta manera, se creará un nuevo campus donde los alumnos, presenciales y virtuales, acceden a materiales diseñados adecuadamente para este doble uso, y donde la interacción profesor-alumno y, sobre todo, alumno-alumno, pueda establecerse con fluidez.

\section{Sistema Integrable de Información de la Universidad Distrital}

Un sistema integrable de información para la Universidad Distrital debe involucrar las diferentes comunidades de usuarios, así como la posibilidad de acceder a la información académica e investigativa al interior de la misma, a nivel Distrital, nacional e internacional.

Esta nueva concepción se basa en la idea de que el sistema puede ser modelado como un conjunto de objetos que interactúan entre sí [Booch, 1996b]. De manera que organizar electrónicamente la información se reduce a identificar dichos objetos y modelar adecuadamente sus características y comportamiento. El aspecto central en el diseño consiste en que todo elemento del sistema real, objeto-real, corresponda directamente a un objeto virtual en el sistema computacional objeto-formal que lo modele [Gellersen, 1999].

De cualquier forma, las nuevas tendencias en el manejo de la información resaltan puntos de importancia tales como la reutilización, compatibilidad, eficiencia, mantenimiento y continuidad del desarrollo informático aplicado.

Los componentes fundamentales tienen que ver con la posibilidad real de la integración de la gestión administrativa, gestión de la educación e investigación y otros sistemas básicos de infraestructura.

La gestión administrativa implica varios sistemas a saber:

- Sistemas de Correo Electrónico y Cartelera Electrónica: proporcionan a profesores, estudiantes y empleados administrativos métodos de acceso y comunicación con el predominio de navegadores. Presentan interfaces amigables que favorecen las iniciativas de trabajo en grupo.

Sistemas de gestión del flujo de trabajo: deberá desarrollarse una interfaz de usuario estandarizada que pueda aplicarse a los diferentes procesos administrativos. Gracias al monitoreo y aprobación de documentos en proceso, este sistema proporciona alta efectividad en los procesos institucionales. 
- $\quad$ Sistemas de gestión de personal: utiliza el modelo de rol especializado para la gestión de datos. Este sistema soporta la gestión digitalizada de los asuntos referentes al personal tales como nomina, capacitación, evaluación, planes de trabajo individual y bibliotecas de fotos del personal.

- Sistemas de gestión financiera: proveen todos los controles e información financiera necesarios para una moderna gestión contable. La base de datos financiera sirve de punto de referencia a otros sistemas tales como los de gestión de la investigación y del flujo de trabajo. La referencia a otros datos del sistema mientras se realiza el procesamiento, en un entorno distribuido, puede considerarse como el primer paso en la integración de sistemas [ISO, 1994a], [ISO, 1994b], [ISO, 1994c].

- $\quad$ Sistema para la seguridad social: permite desarrollar procesos de liquidación de prestaciones sociales afiliación a las EPS, ARP, a fin de normalizar y homogeneizar estos procesos.

La gestión académica busca dotar a los estudiantes y equipos de trabajo con modernos procedimientos para el registro y control académicos. El acceso al registro de cursos debe proporcionarse vía Web e Intranet [Gellersen, 1999], con el propósito de evitar conflictos y cuellos de botella al momento del realizarse los procesos de registro de asignaturas. Debe hacerse investigación en este campo a fin de ofrecer más servicios académicos a través de sistemas de información tales como pago de matrículas, exámenes diferidos y gestión de cuentas de estudiantes en la institución.

El sistema de Gestión del Aprendizaje y la Investigación comprende:

- Sistema de Catálogo de Biblioteca Compartido y en Línea: deberá desarrollarse este sistema para compartir una base de datos bibliográfica única, entre diferentes instituciones, que conducirá a la efectividad en la utilización de los datos y en la reducción de los costos.

- $\quad$ Sistema Estandarizado de Gestión de Información de Biblioteca: se implementará para digitalizar y gestionar toda la información en la Universidad Distrital y otras instituciones y para adquirir dominios de búsqueda más amplios.

Otros sistemas de infraestructura comprenden:

Sistemas de Ventas en Línea: a manera de comercio electrónico, deberá soportar la venta de productos, comparación de precios y factores de calidad y los pedidos en línea.

Periódicos y Revistas Electrónicas: deberá presentar publicaciones relativas a las distintas actividades universitarias.

El sistema de extensión, deberá contar con una base de datos, de consultores, asesores, proyectos de extensión, auditores y redes de extensión.

\section{Conclusiones}

El profundo proceso de reestructuración y transformación institucional que necesita el sector educativo colombiano, en aras de una alta productividad y competitividad, con máxima calidad, requiere un gran esfuerzo donde la calidad e innovación, como sus elementos más críticos, implican además de la participación de las personas más calificadas en el correspondiente campo profesional o disciplinario, la reutilización de la experiencia, teniendo en cuenta su tradición en las distintas áreas del saber y la utilización masiva de las tecnologías de la información, la comunicación y de gestión del conocimiento. 
UNIVERSIDAD PEDAGÓGICA NACIONAL

De esta manera la transformación tecnológica en el sector universitario colombiano, que demanda el mundo contemporáneo, para asumir sus compromisos en la sociedad del conocimiento, requiere de la voluntad real y efectiva de todos sus estamentos, pero también, de audacia en el acopio de recursos tecnológicos, en particular, la masificación del uso de las tecnologías de la información, la comunicación y de gestión del conocimiento.

\section{Bibliografía}

[Booch, 1996a] Booch G., 1996, Análisis y Diseño Orientado a Objetos con Aplicaciones. Addison Wesley, segunda edición.

[Booch, 1996b] Booch G., 1996, Object Solutions. Managing the Object Oriented Project, Addison Wesley.

[Booch, 1999] Booch G, Rumbaugh J., Jacobson I., 1999, El Lenguaje de Modelado Unificado. Addison Wesley .

[Cárdenas, Cárdenas, R. Quiroga, P., Pérez, N., 2001, Documentos de Trabajo, 2001] Red UDNET. Universidad Distrital Francisco José de Caldas,Bogotá.

[D'Souza, 1999] D'Souza D, Cameron A., 1999, Objects, Components and Frameworks with UML. The Catalysis Approach.

[Firestone, Firestone M., 2000, Knowledge Management: A framework for 2000] Analysis and Measurement. October 1, http://www.dkms.com.

[Gellersen, Gellersern, H., 1999, An Object Oriented Model for the Web 1999] Application Development Process, IEEE Internet Computing, January -February.

[Ginige, 2001a] Ginige A., Murugesan A., 2001, Web Engineering: An Introduction. IEEE Multimedia Magazine, January-March.

[Ginige, 2001b] Ginige A., Murugesan A., 2001, The Essence of Web Engineering. Managing the Diversity and Complexity of Web Application Development. IEEE Multimedia Magazine, April-June.

[ISO, 1994a] ISO/IEC CD 10746-1, 1994, Basic Reference Model of Open Distributed Processing - Part 1 : Overview and Guide to Use, July.

[ISO, 1994b] ISO/IEC CD 10746-1, 1994 Basic Reference Model of Open Distributed Processing - Part 2: Descriptive Model, July.

[ISO, 1994c] ISO/IEC CD 10746-1, 1994, Basic Reference Model of Open Distributed Processing - Part 3: Prescriptive Model, July.

[Meyer, 1988] Meyer B., 1998, Object Oriented Software Construction. New York, NY Prentice Hall.

[Nonaka, 1995] Nonaka, I., Takeuchi, H., 1995, The Knowledge Creating Company. Oxford, UK, Oxford University Press.

[Schreiber, Schreiber G., et al., 2000, Knowledge Engineering and Management. 2000]

[Silvio, 2000] The CommonKADS Methodology. The MIT Press, London, England. Silvio, José, 2000, La virtualización de la Universidad, IESALC/UNESCO, Editorial UNESCO, Caracas. 